\title{
Complex Permeability and Permittivity Variation of Radar Absorbing Materials Based on MnZn Ferrite in Microwave Frequencies
}

\author{
Adriana Medeiros Gama*, Mirabel Cerqueira Rezende \\ Divisão de Materiais - AMR, Departamento de Ciência e Tecnologia Aeroespacial-DCTA, Instituto de \\ Aeronáutica e Espaço - IAE, Praça Marechal Eduardo Gomes, 50, Vila das Acácias, CEP 12228-904, \\ São José dos Campos, SP, Brazil
}

Received: July 11, 2012; Revised: February 4, 2013

\begin{abstract}
The complex dielectric permittivity $(\varepsilon)$ and magnetic permeability $(\mu)$ of Radar Absorbing Materials (RAM) based on magnetic particles (MnZn ferrite particles) embedded in a dielectric matrix (silicon rubber) have been studied in the frequency range of 2 to $18 \mathrm{GHz}$. The relative permeability and permittivity of MnZn ferrite-silicon composites for various mass fractions are measured by the transmission/reflection method using a vector network analyzer. The concentration dependence of permittivity and permeability on the evaluated frequency range is analyzed. In a general way, the results show $\varepsilon^{\prime}$ parameter presenting more significant variation among the evaluated parameters ( $\left.\varepsilon^{\prime \prime}, \mu^{\prime \prime}, \mu^{\prime}\right)$. The comparison of dielectric and magnetic loss tangents ( $\varepsilon^{\prime \prime} / \varepsilon^{\prime}$ and $\mu^{\prime \prime} / \mu^{\prime}$, respectively) shows more clearly the variation of both parameters $(\varepsilon$ and $\mu)$ according to the frequency. It is also observed that higher MnZn ferrite content fractions favor both dielectric and magnetic loss tangents.
\end{abstract}

Keywords: MnZn ferrite, silicon, permeability, permittivity, Radar Absorbing Materials (RAM)

\section{Introduction}

Magnetic granular composites consisting of metallic or ceramic magnetic particles embedded in a dielectric matrix have been widely used in electromagnetic applications such as electromagnetic wave absorber (also named Radar Absorbing Materials, RAM) and electromagnetic shielding materials ${ }^{1}$. With the fast advances in wireless communication and defense industries, radar absorbing materials are becoming more and more important in both civilian and military applications, respectively ${ }^{2-4}$. In general, RAM can be divided into two types: dielectric and magnetic ones. For single-layer microwave absorbers that have mainly magnetic losses, in comparison to those that have mainly dielectric losses, broader bandwidth and higher absorption at smaller layer thickness can be achieved ${ }^{5}$. In this way, it can be cited MnZn ferrite as a typical magnetic particle used in the magnetic RAM processing, attending the microwave frequency range. Thus, this magnetic particle has been widely used in the electromagnetic shielding and in RAM processing ${ }^{2,6}$.

Aiming to contribute to this area, this paper shows a study involving the evaluation of complex magnetic permeability $\left(\mu=\mu^{\prime}-\mathrm{j} \mu\right.$ ") and dielectric permittivity $\left(\varepsilon=\varepsilon^{\prime}-\mathrm{j} \varepsilon "\right)$ behaviors of an elastomeric RAM processed with different $\mathrm{MnZn}$ ferrite contents, in the frequency range of 2 to $18 \mathrm{GHz}$.

\section{Material and Methods}

A MnZn ferrite powder supplied by Sontag S.A. Company from Brazil was chosen as microwave absorbing

*e-mail: adrianaamg@iae.cta.br filler. The stoichiometry of the prepared $\mathrm{MnZn}$ ferrite is $\mathrm{Mn}_{0,66} \mathrm{Zn}_{0,34} \mathrm{Fe}_{2} \mathrm{O}_{4}$, which has the spinel structure with a lattice constant $a=8.483 \AA$. A silicon rubber from BASF Ltd. was used as polymeric matrix in the RAM processing. Both components are commercially available. The densities of the employed MnZn ferrite and silicon matrix are 5.03 and $1.28 \mathrm{~g} / \mathrm{cm}^{3}$, respectively. The MnZn ferrite contents into the processed elastomeric RAM were 30, 40, 50, 60 and $70 \%$ in weight concentration (w/w). The elastomeric RAM samples were prepared by conventional mechanical mixture of the two raw materials. The homogeneous mixtures were molded in a coaxial die with inner diameter of $3 \mathrm{~mm}$ and outer diameter of $7 \mathrm{~mm}$. The polymer curing was performed at room temperature for about 24 hours. At the end, flexible cylindrical composite specimens were produced.

Scanning electron microscopy (SEM) examinations were performed employing a DSM950 Zeiss, without special preparation of the samples, and X-ray diffraction (XRD) patterns of $\mathrm{MnZn}$ ferrite powder were obtained using $\mathrm{CuK} \alpha$ radiation from a PW1830 Philips X-ray diffractometer and the diffraction points were recorded from 10 to $90^{\circ}$.

The scattering parameters $\left(S_{11}\right.$ and $S_{21}$ parameters) (Figure 1) ${ }^{7}$ were measured and used to calculate the complex magnetic permeability and dielectric permittivity of all prepared RAM samples. The measurements were performed according to the transmission/reflection method using an HP $8510 \mathrm{C}$ vector network analyzer, adapted with an APC7 coaxial transmission line, in the frequency range of 2 to $18 \mathrm{GHz}$. Figure 1 shows a schematic representation of the measurement system utilized. The Nicolson-Ross modeling was applied in $\mu$ and $\varepsilon$ calculations ${ }^{7}$. 


\section{Results and Discussion}

Figures 2 and 3 show a SEM image and the XRD patterns of the studied MnZn ferrite, respectively. In Figure 2 it is observed various particle sizes $(9-72 \mu \mathrm{m})$ and shapes (acicular and plate) of the evaluated ferrite. The X-ray diffraction pattern for the system $\mathrm{Mn}_{0.66} \mathrm{Zn}_{0.34} \mathrm{Fe}_{2} \mathrm{O}_{4}$ powder (Figure 3 ) shows the existence of a single cubic phase. Table 1 presents the values of $2 \theta$ and the relative intensities of the contributions related to the MnZn ferrite crystal structure. These contributions present good agreement with those obtained by the JCPDS card number 74-2401.

The real (storage) and imaginary (loss) dielectric permittivity ( $\varepsilon^{\prime}$ and $\varepsilon$ ", respectively) and the permeability ( $\mu$ ' and $\mu$ ", respectively) behaviors are shown in Figures 4 and 5 , respectively. Figure 6 shows the dielectric $(\tan \delta \varepsilon=\varepsilon " / \varepsilon ')$ and magnetic $(\tan \delta \mu=\mu$ ' $/ \mu$ ') loss tangents.

Figures $4 \mathrm{a}$ and 5a show that $\varepsilon^{\prime}$ and $\mu$ ' components, respectively, for the neat silicon rubber $(0 \%$ mass concentration) present the lowest values. With the frequency increase the $\mu$ 'values approach to 1.0 and $\varepsilon$ ' varies around 2.6. The imaginary components ( $\mu$ " and $\varepsilon ")$, Figures $4 b$ and $5 \mathrm{~b}$, respectively, present also the lowest values for these parameters (from 0.0 to 0.2 and from 0.0 to 0.1 , respectively). In the same way, the loss tangents present the lowest values (Figure 6). These behaviors mean that the neat silicon matrix presents low magnetic and dielectric
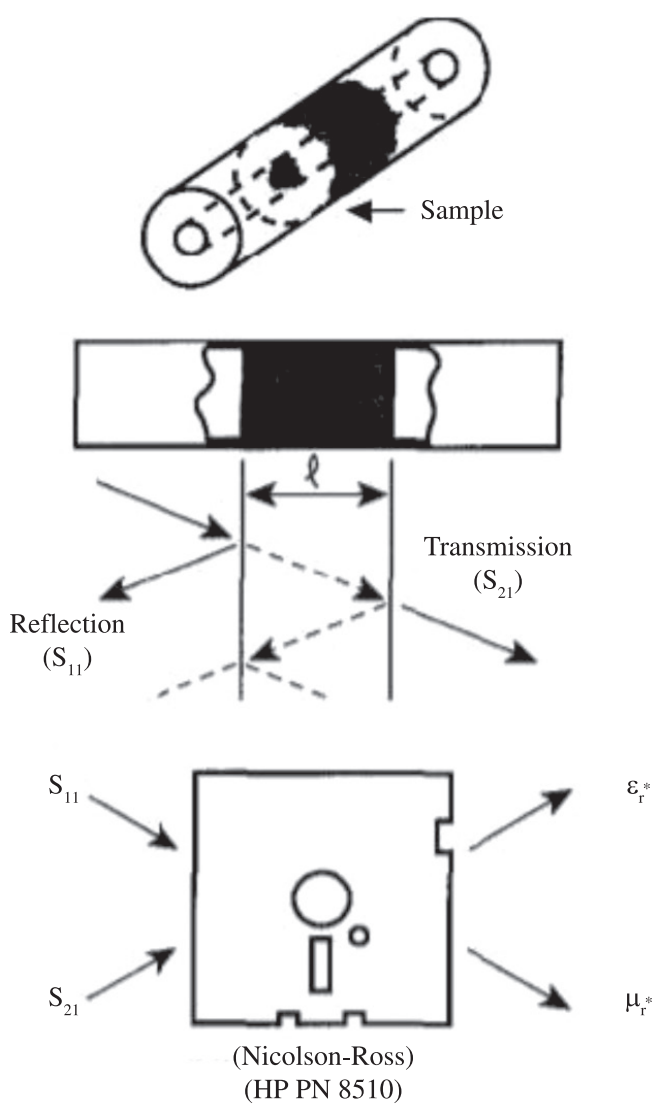

Figure 1. Representation of the transmission/reflection line ${ }^{7}$. losses. The literature cites similar behavior for other pure polymeric matrices ${ }^{4,8,9}$.

Figure 5a shows the real permeability $\left(\mu^{\prime}\right)$ values which present a slight increase with the magnetic filler concentration increase into the RAM sample. For example, at $2 \mathrm{GHz}, \mu$ ' is equal to 1.2 and 1.6 for the samples with 30 and $70 \%(w / w)$ of MnZn ferrite, respectively. On the other hand, this magnetic parameter decreases gradually as the frequency increases. This observation is expected considering the typical behavior of magnetic materials with the frequency increase. It is known that this property decreases with the frequency increase due to the decreasing of both effects domain-wall motion and relaxation effects ${ }^{9,10}$. Similar behavior is also reported by Feng et al. ${ }^{11}$.

In a general way, the imaginary permeability ( $\mu$ ") (Figure 5(b)) presents a similar behavior to that one observed for $\mu^{\prime}$ ' (Figure 5(a)). In this case, it is also observed the imaginary parameter increasing as the magnetic filler concentration increase.

Comparing Figures 4 and 5, it is observed that the real permittivity $\left(\varepsilon^{\prime}\right)$ presents a more accentuated increase with the $\mathrm{MnZn}$ ferrite concentration increase in relation to $\mu$ ' parameter. Moreover, for a same mass fraction, $\varepsilon$ ' parameter keeps almost constant with the frequency variation. The $\varepsilon^{\prime}$ variation with the $\mathrm{MnZn}$ ferrite mass fraction is attributed to the polarization of the dielectric dipoles of the filler into

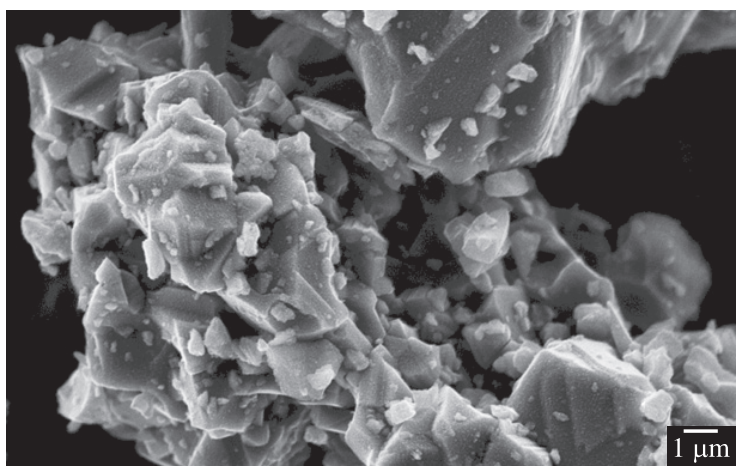

Figure 2. SEM image of the MnZn ferrite used.

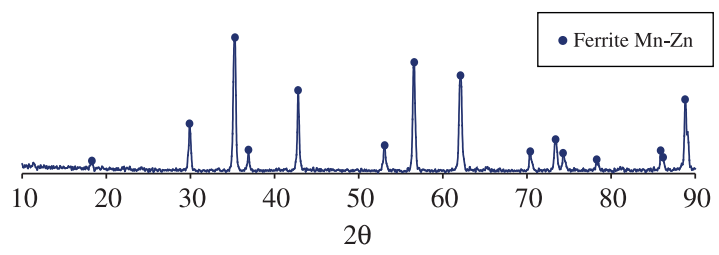

Figure 3. XRD pattern of the $\mathrm{Mn}_{0,66} \mathrm{Zn}_{0,34} \mathrm{Fe}_{2} \mathrm{O}_{4}$ ferrite studied.

Table 1. Typical peaks related to the MnZn phase formation.

\begin{tabular}{cc}
\hline $\boldsymbol{2 \theta} \theta$ & Relative intensity $\left(\mathbf{I} / \mathbf{I}_{\mathbf{0}}\right)$ \\
\hline 35.0 & 100 \\
56.3 & 48 \\
61.8 & 48 \\
29.8 & 28 \\
42.6 & 20 \\
88.7 & 18 \\
\hline
\end{tabular}




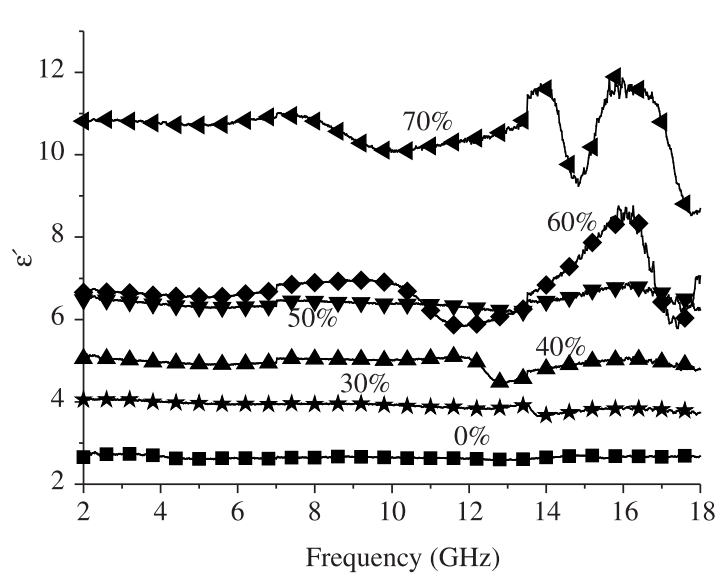

(a)

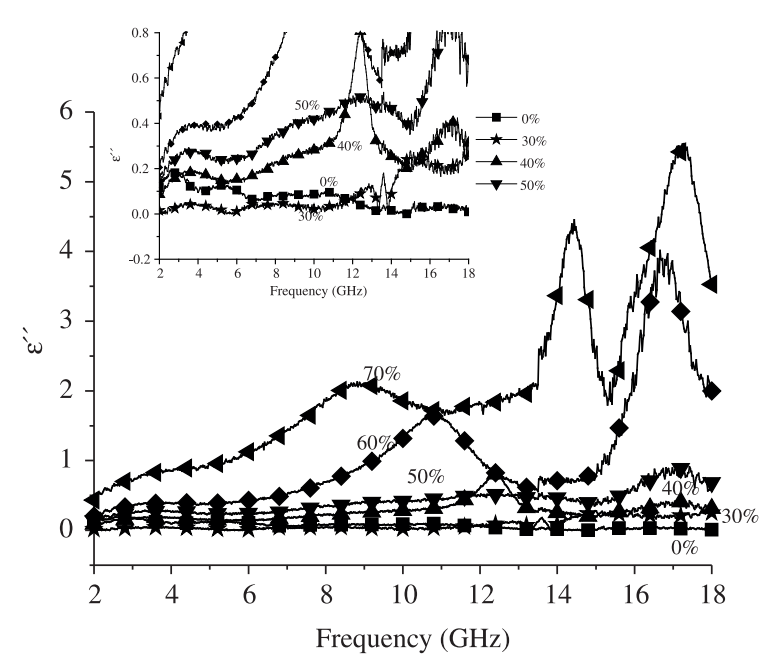

(b)

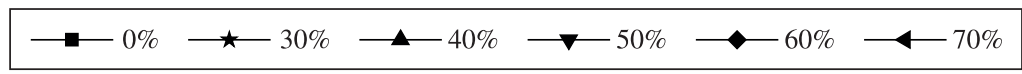

Figure 4. (a) Real and (b) imaginary dielectric permittivity curves for RAM based on MnZn ferrite (0, 30, 40, 50, 60 and 70\% (w/w)) in function of the frequency.

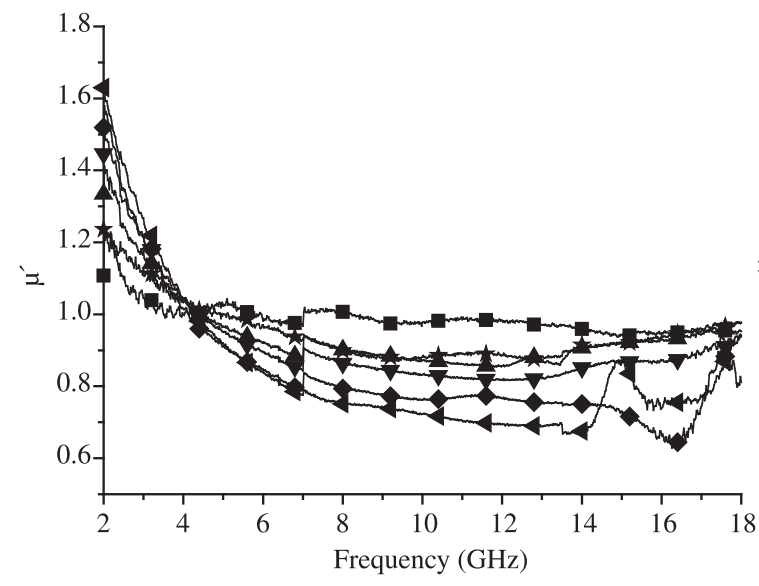

(a)

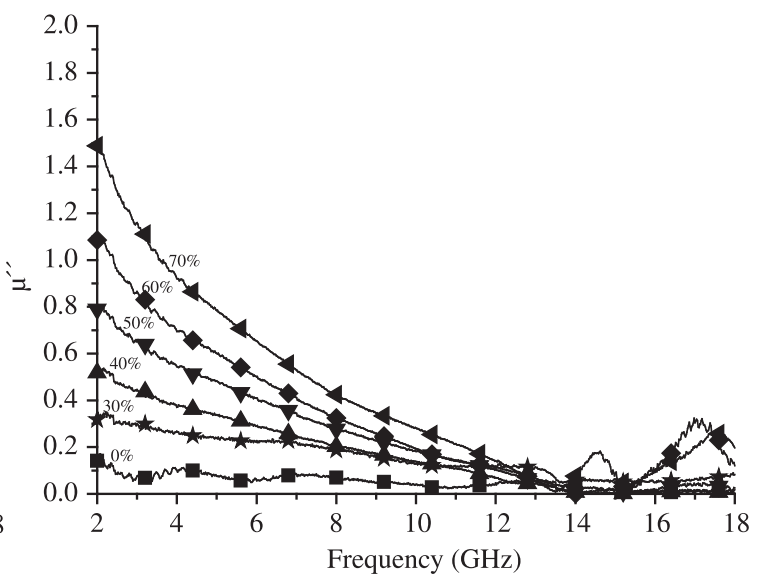

(b)

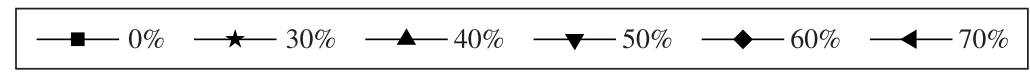

Figure 5. (a) Real and (b) imaginary magnetic permeability curves for RAM based on MnZn ferrite (0, 30, 40, 50, 60 and 70\% (w/w)) in function of the frequency.

the RAM. In this case, it is considered that the dipoles are in-phase with the oscillation of the electric field vector of the electromagnetic wave. On the other hand, the dielectric losses ( $\left.\varepsilon^{\prime \prime}\right)$ increase with the increases of both filler concentration and frequency. This behavior is more accentuated for mass fractions above $60 \%$ and suggests that the energy loss processes during the dipole oscillation, under the electromagnetic wave influence, is more significant for higher frequencies. The discussed behavior is in accordance with Feng et al. ${ }^{11}$.

The dielectric loss tangent ( $\varepsilon^{\prime \prime} / \varepsilon$ ') curves (Figure 6(a)) present a conclusive behavior only for the more concentrated samples $(60$ and $70 \%(\mathrm{w} / \mathrm{w}))$ that show an increase of this parameter concomitant to the frequency increase. Less concentrated samples present, in a general way, values in the range of 0.0 and 0.1 .

The magnetic loss tangent ( $\mu$ '/ $\mu$ ') plots (Figure $6 b$ ) show a slight decrease concomitant to the frequency increase that is attributed to spin inversion losses ${ }^{11}$.

Nelson ${ }^{12}$ reported that the dielectric losses present different loss mechanisms with the frequency increase. When the frequency is relatively low (below $\mathrm{GHz}$ ), the losses are determined mainly by the conductance and are independent of the frequency. Conversely, in frequencies in the microwave range, the losses involve two energy loss mechanisms: polarization-relaxation and electrical 


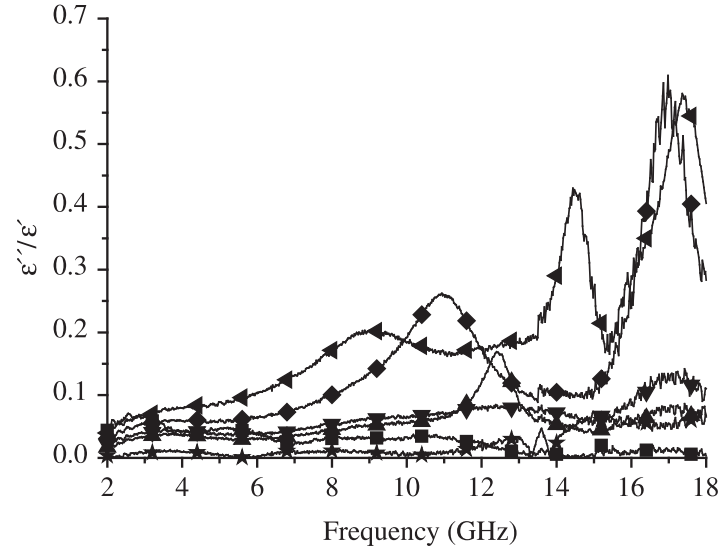

(a)

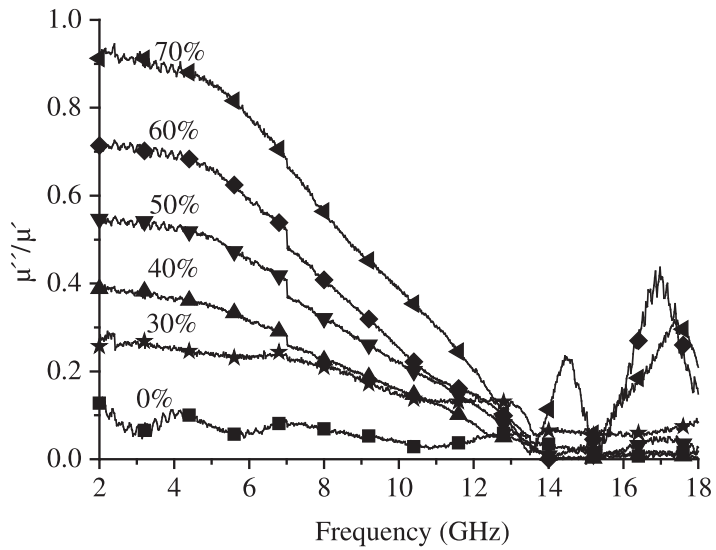

(b)

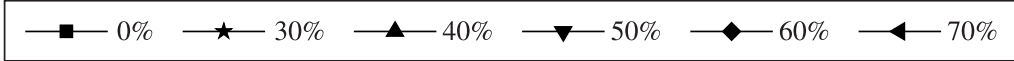

Figure 6. (a) Dielectric and (b) magnetic loss tangents curves for RAM based on MnZn ferrite (0, 30, 40, 50, 60 and 70\% (w/w)) in function of the frequency.

conductance ${ }^{10,12}$. Thus, the two dielectric loss peaks at about 11.0 and $17.0 \mathrm{GHz}$ (Figure 6a) can be attributed to these two loss mechanisms.

The electromagnetic behaviors presented and discussed in this paper show good agreement with the literature, contributing to the processing of RAM and also to the understanding of the wave-matter interaction involved in this area. However, it is important to mention that changes in morphological and structural characteristics of the ferrites can promote differences on the electromagnetic behavior of RAM. Thus, the SEM and X-ray diffraction analyses presented in this study are important to support other comparisons in future studies.

\section{Conclusion}

The results of this study involving the electromagnetic properties of radar absorbing materials based on $\mathrm{MnZn}$ ferrite/rubber show the dependence of both complex magnetic permeability $(\mu)$ and dielectric permittivity $(\varepsilon)$ parameters on the frequency, in the range of 2 to $18 \mathrm{GHz}$.

\section{References}

1. Park MJ, Choi J and Kim SS. Wide bandwidth pyramidal absorbers of granular ferrite and carbonyl iron powders. IEEE Transactions on Magnetics. 2000; 36(5):3272-3274. http:// dx.doi.org/10.1109/20.908766

2. Liu AX, Ru M, Meng F, Zhang W, Zhang Q and Zhou Q. Preparation and microwave absorbing property of nanosize PSZFe magnetic particles. Acta Polymerica Sinica. 2003; (5):757-760.

3. Feng YB, Qiu T and Shen CY. Absorbing properties and structural design of microwave absorbers based on carbonyl iron and barium ferrite. Journal of Magnetism and Magnetic Materials. 2007; 318(1-2):8-13. http://dx.doi.org/10.1016/j. jmmm.2007.04.012
Firstly, the complexity involving the behavior of these parameters in the microwave frequency range is observed. It is also observed a significant variation of $\varepsilon$ ' among the evaluated parameters ( $\varepsilon^{\prime \prime}, \mu$ ', $\mu$ ') with the MnZn ferrite concentration. The comparison of dielectric and magnetic loss tangents ( $\varepsilon$ " $/ \varepsilon$ ' and $\mu$ '/ $\mu$ ', respectively) shows more clearly the variation of both parameters $(\varepsilon$ and $\mu)$ with the frequency. It is also observed that higher MnZn ferrite content fractions favor both dielectric and magnetic loss tangents. Finally, based on the literature data, it is possible to suggest that the dielectric loss mechanisms for RAM, in the microwave range, involve polarization-relaxation phenomenon, electric conductance and spin inversion for magnetic losses.

\section{Acknowledgements}

The authors acknowledge the financial assistance of Financiadora de Estudos e Projetos (FINEP) (Process number 1757-6) and the National Council for Research and Development (CNPq) (Process number 305478/09-5).

4. Yusoff AN, Abdullah MH, Ahmad SH, Jusoh SF, Mansor AA and Hamid SAA. Electromagnetic and absorption properties of some microwave absorbers. Journal of Applied Physics. 2002; 92(2):876-882. http://dx.doi. org/10.1063/1.1489092

5. Giannakopouou T, Kontogeorgakos A and Kordas G. Singlelayer Microwave absorbers: influence of dielectric and magnetic losses on the layer thickness. Journal of Magnetism and Magnetic Materials. 2003; (263):173. http://dx.doi. org/10.1016/S0304-8853(02)01551-2

6. Yong $\mathrm{W}$, Afsat $\mathrm{MN}$ and Grignon R. Complex permittivity and permeability of carbonyl iron powders at microwave frequencies. IEEE Antennas and Propagation Society International Symposium. 2003; (4):619-622. 
7. Nicolson AM and Ross GF. Measurement of the Intrinsic Properties of Materials by Time Domain Techniques. Instrumentantion and Measurement. 1970; 19:377-382. http:// dx.doi.org/10.1109/TIM.1970.4313932

8. Naito Y and Suetake K. Application of ferrite to electromagnetic wave absorber and its characteristics. IEEE Microwave Theory and Techniques Society . 1971; 19(1):65-72. http://dx.doi. org/10.1109/TMTT.1971.1127446

9. Gama AM, Rezende MC and Dantas CC. Dependence of microwave absorption properties on ferrite volume fraction in MnZn ferrite/rubber radar absorbing materials. Journal of Magnetism and Magnetic Materials. 2011; 323(22):2782-2785. http://dx.doi.org/10.1016/j.jmmm.2011.05.052
10. Gama AM and Rezende MC. Complex permeabillity and permittivity variation of carbonyl iron rubber in the frequency range of 2 to $18 \mathrm{GHz}$. Journal of Aerospace Technology and Management. 2010; 2(1):59-62. http://dx.doi.org/10.5028/ jatm.2010.02015962

11. Feng Y-B, Qiu, T, Shen C-Y and Li X-Y. Electromagnetic and absorption properties of carbonyl iron/rubber radar absorbing materials. IEEE Transactions on Magnetics. 2006; 42(3):363-368. http://dx.doi.org/10.1109/TMAG.2005.862763

12. Nelson SO. Density-permittivity relationships for powdered and granular materials. IEEE Transactions on Instrumentation and Measurement. 2005; 54(5):2033-2040. http://dx.doi. org/10.1109/TIM.2005.853346 